\title{
Discovery of VHE Gamma-Ray Emission from the Binary System LMC P3
}

\author{
Nukri Komin* \\ School of Physics, University of the Witwatersrand, Johannesburg, South Africa. \\ E-mail: nukri.komin@wits.ac.za
}

\section{Maria Haupt}

DESY, Zeuthen, Germany.

\section{for the H.E.S.S. Collaboration ${ }^{\dagger}$}

\begin{abstract}
Recently, the $\gamma$-ray emission at $\mathrm{MeV}$ and $\mathrm{GeV}$ energies from the object LMC P3 in the Large Magellanic Cloud has been discovered to be modulated with a 10.3-days period, making it the first extra-galactic $\gamma$-ray binary. This work aims at the detection of $\mathrm{TeV} \gamma$-ray and the search for modulation of the signal with the orbital period of the binary system. The H.E.S.S. data set has been folded with the known orbital period of the system in order to test for variability of the emission. Energy spectra are obtained for the orbit-averaged data set and for orbital phases in which the $\mathrm{TeV}$ flux is found at its maximum. $\mathrm{TeV} \gamma$-ray emission is detected with a statistical significance of $6.4 \sigma$. The data clearly show variability which is phase-locked to the orbital period of the system. Periodicity cannot be deduced from the H.E.S.S. data set alone. The orbit-averaged luminosity in the $1-10 \mathrm{TeV}$ energy range is $(1.4 \pm 0.2) \times 10^{35} \mathrm{erg} \mathrm{s}^{-1}$. A luminosity of $(5 \pm 1) \times$ $10^{35} \mathrm{erg} \mathrm{s}^{-1}$ is reached during $20 \%$ of the orbit, when the $\mathrm{MeV} / \mathrm{GeV}$ emission is at its minimum.
\end{abstract}

35th International Cosmic Ray Conference - ICRC2017

10-20 July, 2017

Bexco, Busan, Korea

\footnotetext{
${ }^{*}$ Speaker.

${ }^{\dagger}$ https://www.mpi-hd.mpg.de/hfm/HESS/pages/collaboration/
} 


\section{Introduction}

More than $60 \%$ of all stellar systems containing high-mass stars (spectral type B2 or earlier) are binary or multiple systems [1]. When the more massive of the stars in these systems ends its life in a supernova explosion, a binary system is left behind where a compact object, either a neutron star or a black hole, is orbiting the remaining star. Matter accretion from the star to the compact object may lead to X-ray emission. These objects are known as X-ray binaries. If the companion is also a massive star, the system is called a high-mass X-ray binary (HMXB). When the emission of these objects dominates in the $\gamma$-ray regime (high-energy (HE) $\gamma$ rays: $0.1-100 \mathrm{GeV}$, very-highenergy (VHE) $\gamma$ rays: $>100 \mathrm{GeV}$ ) then they are called $\gamma$-ray binaries. Up to now only six $\gamma$-ray binaries are known. The $\gamma$-ray emission arises either from the interaction of the pulsar and stellar winds or from accretion onto a black hole or neutron star. The companion stars in these systems are either O-type or Be stars. The nature of the compact objects are generally unknown, with the exception of PSR B1259-63/LS 2883 where the detection of pulsed emission [2] shows that the compact object is a neutron star. A review of $\gamma$-ray binaries and their properties is given by [3, 4].

In order to identify previously undetected $\gamma$-ray binaries, [5] performed a blind search for periodic emission in the Fermi-LAT data. They found that the $\mathrm{MeV} / \mathrm{GeV} \gamma$-ray signal of LMC P3 is periodic with a period of $10.301 \pm 0.002$ days. These authors defined phase 0 of this system to correspond to the maximum in the gamma-ray emission at MJD 57410.25 \pm 0.34 . LMC P3 is a previously unidentified $\gamma$-ray source [6] located in the Large Magellanic Cloud (LMC). The position of LMC P3 is consistent with the position of CAL 60, which is a soft X-ray source discovered by [7]. [8] identify a star of spectral type O5 III(f) as likely counterpart of this X-ray source. Subsequent X-ray observations with XMM-Newton [9] and Chandra [10] confirm a point-like X-ray source which is named CXOU 053600.0-673507. [10] already concluded from the variabilities of the X-ray flux and the radial velocity of Balmer absorption lines that this object is likely a binary system. LMC P3 is located in the supernova remnant DEM L241, making it the third X-ray binary found in an observable supernova remnant after SS433/W 50 [11] and SXP 1062 [12].

Little is known of the orbital parameters of the system. The most precise measurement of the orbital period comes from the HE $\gamma$-ray emission. [5] also analysed the radial velocity of the star and found an orbital period of 10.1 days and a superior conjunction of the companion star at MJD $57408.61 \pm 0.28$. The mass function prefers a neutron star as compact object for a wide range of inclinations, but a black hole cannot be ruled out. The X-ray and radio emission of this object is modulated with the 10.3-days period, but is out of phase with the $\gamma$-ray emission [5].

Showing periodic $\gamma$-ray emission from a system with an $\mathrm{O} 5$ companion star makes this object clearly a high-mass $\gamma$-ray binary. It is the first such object discovered outside the Milky Way. With a $\gamma$-ray luminosity in the energy range from $200 \mathrm{MeV}$ to $100 \mathrm{GeV}$ of $2.5 \times 10^{36} \mathrm{erg} \mathrm{s}^{-1}$ [6] it is also the most luminous $\gamma$-ray binary known so far.

\section{H.E.S.S. Observations and Results}

The LMC has been observed extensively with the High Energy Stereoscopic System (H.E.S.S.) since 2004. These observations lead to the discovery of three individual $\mathrm{TeV} \gamma$-ray emitting sources $[13,14]$. H.E.S.S. is a system of five Imaging Cherenkov Telescopes, located in the Khomas 


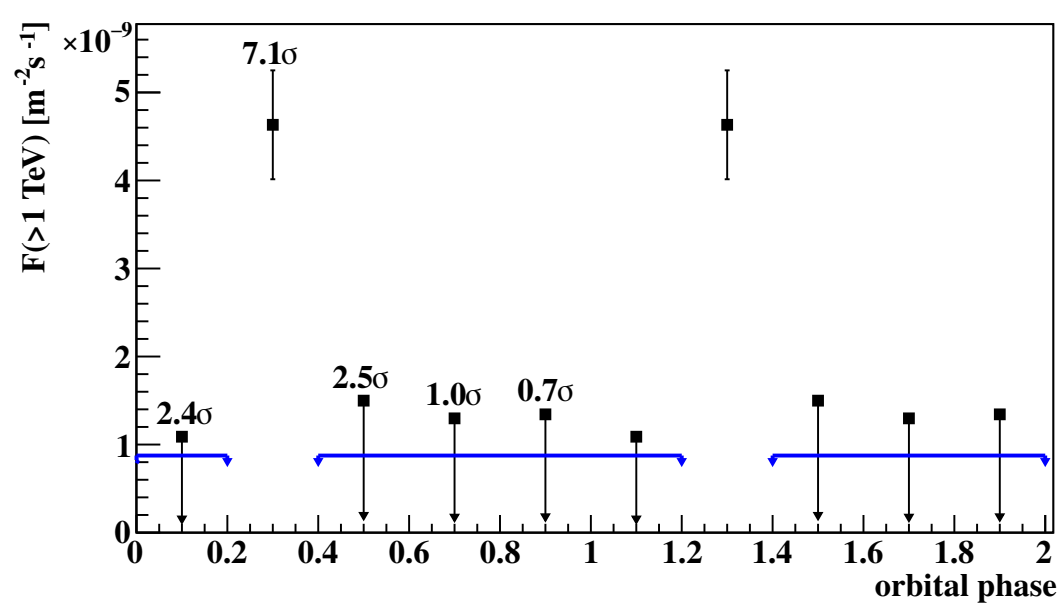

Figure 1: Folded TeV $\gamma$-ray light curve. Orbital phase 0 is defined at the maximum of the Fermi-LAT emission at MJD 57410.25. For better readability two orbits are shown. The flux is computed in orbital phase bins of equal size and similar exposure. Error bars represent $1 \sigma$ statistical uncertainty. For the phase bins without significant detection upper limits (at 95\% confidence level) are given. The labels at the data points indicate the statistical significance of the excess in each phase bin. The blue lines denote the upper limit (at 95\% confidence level) on the flux in the off-peak region (orbital phase 0.4 to 0.2 ).
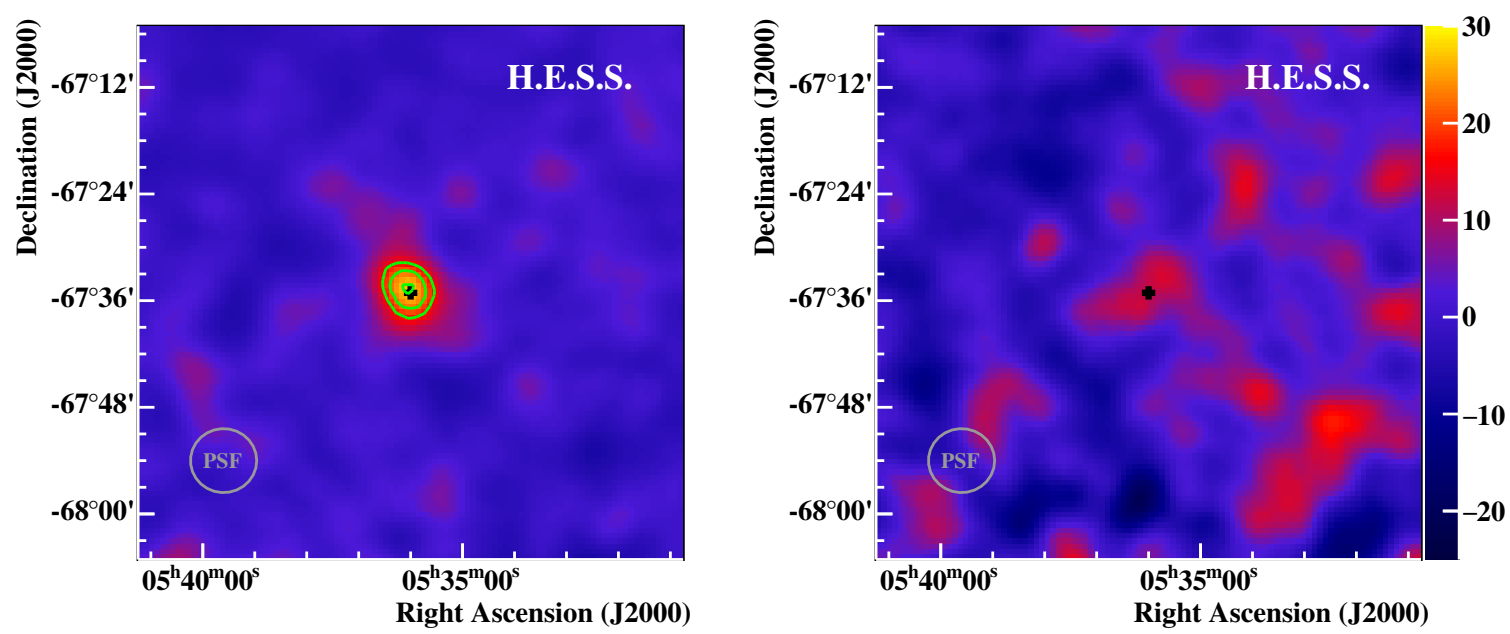

Figure 2: H.E.S.S. excess maps for the on-peak (left panel) and off-peak (right panel) regions of the orbit. The excess is smoothed with the point-spread function of the instrument $(68 \%$ containment radius of $0.06 \mathrm{deg}$, indicated by the grey circle). The cross indicates the test position. Both plots have the same range (colour bar on the right-hand side). The overlaid green contours represent 4, 5 and $6 \sigma$ statistical significance. The on-peak region covers orbital phases from 0.2 to 0.4 , the off-peak region the orbital phases from 0.4 to 0.2 . Orbital phase 0 is defined at the maximum of the Fermi-LAT emission at MJD 57410.25. 
Table 1: Statistical results and spectral parameters for different orbital phase bins of HESS J0536-675. The on-peak region covers orbital phases from 0.2 to 0.4 , the off-peak region the orbital phases from 0.4 to 0.2 . Orbital phase 0 is defined at the maximum of the Fermi-LAT emission at MJD 57410.25.

\begin{tabular}{lcccc}
\hline \hline orbital phase bin & $\begin{array}{c}\Phi_{1 \mathrm{TeV}} \\
{\left[10^{-13} \mathrm{~cm}^{-2} \mathrm{~s}^{-1} \mathrm{TeV}^{-1}\right]}\end{array}$ & $\Gamma$ & $\begin{array}{c}F(>1 \mathrm{TeV}) \\
{\left[10^{-13} \mathrm{~cm}^{-2} \mathrm{~s}^{-1}\right]}\end{array}$ & $\begin{array}{c}L(1-10 \mathrm{TeV}, 50 \mathrm{kpc}) \\
{\left[10^{35} \mathrm{erg} \mathrm{s}^{-1}\right]}\end{array}$ \\
\hline full orbit & $2.0 \pm 0.4$ & $2.5 \pm 0.2$ & $1.4 \pm 0.4$ & $1.4 \pm 0.2$ \\
on peak & $5 \pm 1$ & $2.1 \pm 0.2$ & $5 \pm 2$ & $5 \pm 1$ \\
off peak & - & 2.4 (fixed) & $<1.2(95 \% \mathrm{CL})$ & $<1.2(95 \% \mathrm{CL})$ \\
\hline \hline
\end{tabular}

Highland of Namibia at an altitude of $1800 \mathrm{~m}$. Located in the southern hemisphere it is currently the only instrument for TeV observations of the LMC. Phase I of H.E.S.S. is sensitive to $\gamma$ rays from energies above $100 \mathrm{GeV}$ up to several tens of $\mathrm{TeV}$. The arrival direction of individual $\gamma$ rays can be reconstructed with an angular resolution of better than $0.1^{\circ}$, and their energy is estimated with an relative uncertainty of $15 \%$. The data presented here were taken between 2004 and beginning of 2016 and add up to a total observation time of $277 \mathrm{~h}$, almost $70 \mathrm{~h}$ more than what was used in the previous publication [14]. The earlier observations were centred on the region around N 157B resulting in relatively large camera offsets for LMC P3. In the last years the observation strategy has been optimised in order to obtain a better exposure on LMC P3. After correcting for dead time and camera offset angles the effective exposure time is $100 \mathrm{~h}$. In some of the observation runs the large H.E.S.S.-II telescope was present. The data recorded with this telescope are ignored in the analysis in order to obtain a homogeneous data set. The data were analysed using Model analysis with high-resolution cuts [15], where the camera images are compared with simulations using a log-likelihood minimisation. The background was estimated from rings around each sky position to generate the gamma-ray image [16] and from test regions with similar offsets from the camera centre for the spectral analysis [16]. The energy threshold for this data set is $714 \mathrm{GeV}$.

At the nominal position of CXOU 053600.0-673507 an excess of $76.3 \gamma$ rays is detected with a statistical significance of $6.4 \sigma$ (Table 1 ). The nightly light curve of the emission does not show any sign of variability. The search for periodic emission using a Lomb-Scargle test [16, 17] and the Z-Transformed Discrete Correlation Function [17] does not show any sign of periodicity. The sensitivity of H.E.S.S. does not allow a detection of the object on a nightly basis, which is preventing the detection of variability and periodicity in the nightly light curve. Figure 1 shows the light curve folded with the orbital period of the system of 10.301 days, where orbital phase 0 is defined at the maximum of the Fermi-LAT light curve at MJD 57410.25 [5]. Significant emission is detected only at an orbital phase between 0.2 and 0.4 with a pre-trial significance of $7.1 \sigma$ (corresponding to $6.9 \sigma$ post-trial after 5 trials). All other phase bins do not show any emission (significances less than $2.5 \sigma$, see Fig. 1). All phase bins have roughly the same exposure (between 18 and 21 hours). The fit of a constant to the flux values in the orbital phase bins results in a $\chi^{2}$ value of 27.03 for 4 degrees of freedom. The $\chi^{2}$ probability that the folded light curve is constant is less than $1.95 \times 10^{-5}$. The emission is clearly variable and it is phase-locked to the orbital period of the system. Therefore, the detected $\mathrm{TeV} \gamma$-ray emission can be associated to the binary system LMC P3.

Figure 3 shows the energy spectra for the full orbit and the on-peak part of the orbit. The 


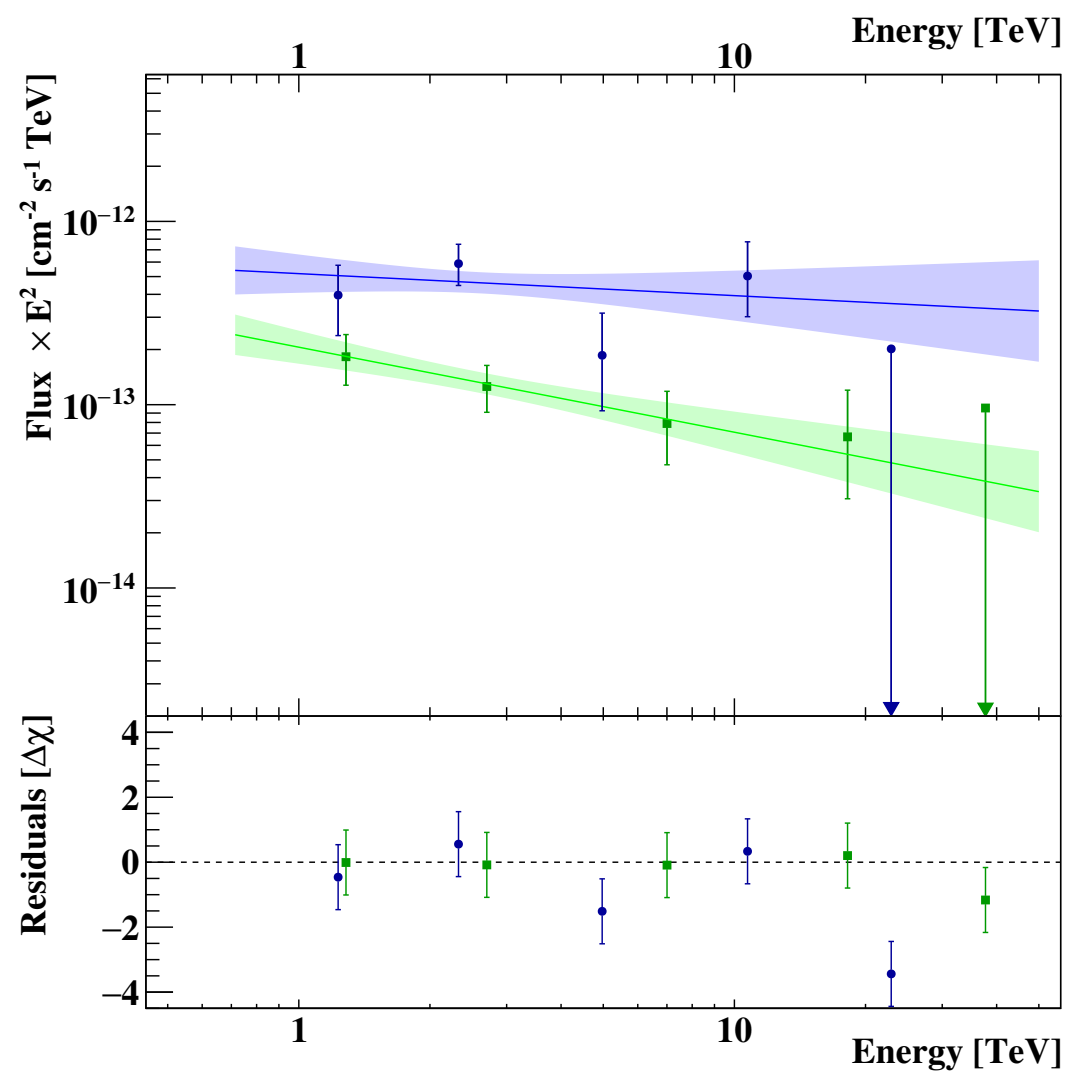

Figure 3: Spectral energy distribution averaged over the full orbit (green, square points) and for the on-peak orbital phase range (orbital phase from 0.2 to 0.4 : blue, circle points). The data points have $1 \sigma$ statistical error bars, upper limit are for a $95 \%$ confidence level. The best fit and its uncertainty are represented by the solid lines and shaded areas, respectively.

spectra are fitted with a simple power law, $\frac{d N}{d E}=\Phi_{1 \mathrm{TeV}}\left(\frac{E}{1 \mathrm{TeV}}\right)^{-\Gamma}$, and the best-fit parameters are summarised in Table 1. No high-energy cut-off of the spectra has been detected. For the off-peak region of the orbit an upper limit on the integrated $\gamma$-ray flux has been obtained. Most of the $\gamma$-ray emission is radiated during only $20 \%$ of the binary's orbit, when it reaches a flux of about 4 times the orbit-averaged flux.

\section{Discussion}

With an on-peak luminosity of $(5 \pm 1) \times 10^{35} \mathrm{erg} \mathrm{s}^{-1}$ LMC P3 is by far the most luminous $\gamma$ ray binary. Two main scenarios are usually invoked to explain the $\gamma$-ray emission from gamma-ray binaries: particle acceleration in a pulsar wind nebula (PWN) driven by the spin-down of the pulsar or accretion of the stellar wind on the compact object.

The $\gamma$-ray luminosity is about the same as the luminosity of the PWN N 157B in the LMC. N 157B is driven by the spin-down of its central pulsar PSR J0537-6910 with a spin-down luminosity of $4.9 \times 10^{38} \mathrm{erg} \mathrm{s}^{-1}$. A putative pulsar in LMC P3 would need about the same spin-down luminosity. This would make it one of the four most luminous pulsars. That such a luminous 
pulsar remains undetected so far can be explained by absorption in the stellar photon field of the companion star.

In an alternative scenario stellar wind is accreted on the compact object. O-type stars have a mass loss through their stellar wind of at least $10^{-6} M_{\odot} \mathrm{year}^{-1}$, corresponding to $6 \times 10^{40} \mathrm{erg} \mathrm{s}^{-1}$. Therefore a fraction of about $10^{-5}$ of the acceretion power needs to be converted into $\gamma$ rays. It should be noted that in such a scenario the formation of jets may be expected (microquasars) which are usually detected at radio energies. No such jets are known for LMC P3.

While the nature of the compact object and the mechanism that leads to the $\gamma$-ray emission are not yet clear, it can already be said that a very luminous pulsar or a strong stellar wind is necessary to provide the energy for the observed $\gamma$-ray emission.

In order to better understand the binary system optical observations to measure the orbital parameters are necessary. Furthermore, a deeper characterization of the system at VHEs will only come with the future Cherenkov Telescope Array (CTA), which will provide the required sensitivity to measure the relatively faint gamma-ray emission produced during the off-peak parts of the orbit.

\section{Summary}

Significant $\mathrm{TeV} \gamma$-ray emission has been detected from the binary system LMC P3 with H.E.S.S.With an O-type companion star this system is similar to LS 5039 and 1FGL J1018.6-5856. The relatively peaky light-curve is similar to 1FGL J1018.6-5856, but GeV and $\mathrm{TeV}$ emission are in anti-phase as is the case for LS 5039. The on-peak luminosity of $(5 \pm 1) \times 10^{35} \mathrm{erg} \mathrm{s}^{-1}$ makes it by far the most luminous $\gamma$-ray binary. A pulsar with a large spin-down luminosity of about $10^{38} \mathrm{erg} \mathrm{s}^{-1}$ or a strong stellar wind are necessary to provide for the energy in $\gamma$-ray photons.

\section{References}

[1] Duchêne, G. \& Kraus, A. 2013, ARA\&A, 51, 269

[2] Johnston, S., Manchester, R. N., Lyne, A. G., et al. 1992, ApJ, 387, L37

[3] Dubus, G. 2013, A\&A Rev., 21, 64

[4] Dubus, G. 2015, Comptes Rendus Physique, 16, 661

[5] Corbet, R. H. D., Chomiuk, L., Coe, M. J., et al. 2016, ApJ, 829, 105

[6] Ackermann, M., Albert, A., Atwood, W. B., et al. 2016, A\&A, 586, A71

[7] Long, K. S., Helfand, D. J., \& Grabelsky, D. A. 1981, ApJ, 248, 925

[8] Crampton, D., Cowley, A. P., Thompson, I. B., \& Hutchings, J. B. 1985, AJ, 90, 43

[9] Bamba, A., Ueno, M., Nakajima, H., Mori, K., \& Koyama, K. 2006, A\&A, 450, 585

[10] Seward, F. D., Charles, P. A., Foster, D. L., et al. 2012, ApJ, 759, 123

[11] Dubner, G. M., Holdaway, M., Goss, W. M., \& Mirabel, I. F. 1998, AJ, 116, 1842

[12] Hénault-Brunet, V., Oskinova, L. M., Guerrero, M. A., et al. 2012, MNRAS, 420, L13

[13] H.E.S.S. Collaboration, Abramowski, A., Acero, F., et al. 2012, A\&A, 545, L2

[14] H.E.S.S. Collaboration, Abramowski, A., Aharonian, F., et al. 2015, Science, 347, 406 
[15] de Naurois, M. \& Rolland, L. 2009, Astroparticle Physics, 32, 231

[16] Lomb, N. R. 1976, Ap\&SS, 39, 447

[17] Scargle, J. D. 1982, ApJ, 263, 835

[18] Berge, D., Funk, S., \& Hinton, J. 2007, A\&A, 466, 1219

[19] Alexander, T. 1997, in Astrophysics and Space Science Library, Vol. 218, Astronomical Time Series, ed. D. Maoz, A. Sternberg, \& E. M. Leibowitz, 163 